\title{
Electro-Rheological Response of Anisotropic Solution of Poly(hexyl isocyanate) Measured by Parallel Plates Sliding Rheometer
}

\author{
Katsufumi Tanaka, Ryuichi AkiYama, and Keigo Takada \\ Department of Polymer Science and Engineering, \\ Kyoto Institute of Technology, Matsugasaki, Kyoto 606, Japan
}

(Received September 25, 1995)

\begin{abstract}
The electro-rheological (ER) response of an anisotropic solution of a commercially available poly(hexyl isocyanate) (PHIC) in p-xylene with a concentration of $30 \mathrm{wt} \%$ was measured at a shear rate of $2.56 \mathrm{~s}^{-1}$ by our parallel plates sliding rheometer. The solution was deformed at the shear rate to be a steady flow, and subsequently it was stimulated by step-wise electric fields with amplitudes up to $3.0 \mathrm{kV} \mathrm{mm}^{-1}$. In the transient ER response, the stress overshoot was observed at electric fields of $1.0 \mathrm{kV} \mathrm{mm}^{-1}$ as well as $1.5 \mathrm{kV} \mathrm{mm}^{-1}$. The transient stress tended to approach to a steady stress depending on the electric field strength although the steady shear stress could not be necessarily measured by the sliding rheometer which limited the maximum shear strain. The maximum shear stress enhanced by the electric fields in the present experiment was in excess of 20 times the steady shear stress under no electric field. The ER response was discussed in terms of the orientation of the PHIC molecules induced by the external electric fields.

KEY WORDS Electro-Rheology / Polymer Solutions / Liquid Crystals / Poly(hexyl isocyanate) / Sliding

Rheometer /
\end{abstract}

The electro-rheological (ER) fluids are known as the fluids which show the large increase in apparent viscosity and recovery to the original viscosity by application and removal of an external electric field, respectively. In these ER fluids, the heterogeneous fluids of suspensions composed of polarizable particles and insulating solvents are widely known as typical ER fluids. The ER suspensions have been investigated extensively by many researchers ${ }^{1-3}$ mainly because of the remarkable increase in the apparent viscosity of several hundreds times and the fast rheological response on a time scale of milliseconds. However, the ER suspensions have the problem of sedimentation of dispersed particles in nature which leads to the instability of the ER effect for a long period in a practical use. Recently, the stability of the ER suspensions has been improved to a certain extent by the precise control of size of particles, the use of surfactant which covers the surface of particles and so on. ${ }^{4,5}$

There are other ER fluids of homogeneous system such as liquid crystals of small molecules ${ }^{6-8}$ and solutions of flexible polymers with polar side group. ${ }^{9}$ In nature, the homogeneous fluids are free from the problem of the sedimentation of particles, which seems to be a favorable feature to a practical purpose. Although the enhancement of the apparent viscosity by the electric fields for the homogeneous fluids as mentioned above is much smaller, typically several times, than that of the ER suspensions, intense interest has been shown toward the homogeneous fluids of liquid crystalline polymers with mesogenic side group ${ }^{10}$ because of the comparable enhancement of apparent viscosity to the ER suspensions. However, it is still unclear the mechanism of the ER effect of these homogeneous fluids. For instance, the ER effect of liquid crystals composed of small and rod-like molecules is explained mainly by the Miesowicz viscosities as shown in Figure 1 as well as the electrohydrodynamic instability ${ }^{6,7}$ which is accompanied by the secondary flow and is caused by the electrical con- duction of charge carriers from electrodes. However, the ER effect of the other homogeneous fluids based on polymers cannot be explained simply by the Miesowicz viscosities and the electro-hydrodynamic instability. Although the ER effect of the polymer based fluids is supposed to be closely related to the molecular entanglements among the polymer main chains or the interaction among side chains, it seems to be difficult to clarify the mechanism of the ER effect directly because of the complex feature of the flexibility of polymer chains. To simplify the problem, it is effective to investigate the ER behavior of the main chain type liquid crystalline polymers composed of rod-like or helical molecules with large aspect ratio such as poly $(\gamma \text {-benzyl-L-glutamate })^{11}$ and poly(hexyl isocyanate $)^{12}$ (PHIC), the latter of which is the subject in the present paper.

From the extensive investigations such as a number of isotropic solutions of PHIC with narrow molecular weight distribution in toluene ${ }^{13-15}$ and in hexane, ${ }^{16}$ it is considered that isotropic solutions of PHIC are well characterized and that PHIC is a typical semi-flexible polymer in the isotropic phase. On the other hand, it is well assumed in the investigation on the ER properties of an anisotropic solution that PHIC rigidity with a narrow distributed weight average molecular weight of $1.8 \times 10^{5}$ would be enhanced in a nematic phase in $p$-xylene. ${ }^{12}$ Further, PHIC, which is a helical polymer, has a large permanent dipole moment along the helix

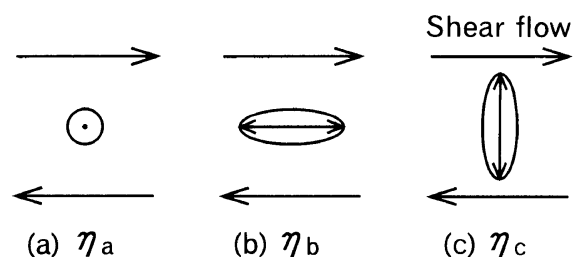

Figure 1. Miesowicz viscosities ${ }^{20}$; (a) director perpendicular to flow direction and to velocity gradient, (b) director parallel to flow direction, and (c) director parallel to velocity gradient $\left(\eta_{\mathrm{a}}, \eta_{\mathrm{b}}<\eta_{\mathrm{c}}\right)$. 
axis ${ }^{17}$ and the induced dipole moment is negligible. ${ }^{18} \mathrm{It}$ is considered that the PHIC molecules tend to orient so that the helix axes orient parallel to the external electric fields and the orientation transverse to the flow direction can enhance the flow resistance ${ }^{12}$ : The ER effect of the anisotropic solution composed of large and rodlike molecules can follow the mechanism of Miesowicz viscosities, and the electro-hydrodynamic instability is expected to be negligible for the large and rod-like molecules.

In the present paper, the ER response is reported for an anisotropic solution of a commercially available PHIC with a broad molecular weight distribution in $p$-xylene measured at a constant shear rate by our parallel plates sliding rheometer and the ER response is discussed in terms of the orientation of the helical molecules induced by the external electric fields.

\section{EXPERIMENTAL}

Poly(hexyl isocyanate) was purchased from General Science Corporation. The molecular weight distribution of the PHIC dissolved in chloroform at room temperature was characterized by GPC using a polystyrene standard and the universal calibration. The polydispersity index $M_{w} / M_{n}$ was 66 with a broad distribution of the molecular weight. The weight average molecular weight was reduced to be $6.9 \times 10^{4}$ using the polystyrene standard. The PHIC as received was dissolved in $p$ xylene. The concentration of the solution was $30 \mathrm{wt} \%$, and the solution was found to be anisotropic at the concentration and room temperature from the optical observations as mentioned in the next paragraph.

Texture of the solution in a quiescent state was observed by an optical microscope (OLYMPUS, BH-2) with crossed polarizers. In the present experiment, all measurements were performed at room temperature. The orthoscopy as well as conoscopy was performed directly in the microscope. A glass plate, one surface of which was covered with tin-doped indium oxides (ITO), was used as a transparent electrode. The solution was sandwiched between two electrodes separated by poly(ethylene terephthalate) spacer films with $25 \mu \mathrm{m}$ thick and connected to a DC power supply. External electric fields up to $1.6 \mathrm{kV} \mathrm{mm}^{-1}$ were biased to the solution using the DC power supply. Further, some glass plates were coated with a silane coupler (Toray Silicone, AY43) and they were used for electrodes as well. It is well known that liquid crystals of small molecules preferentially align perpendicular to the glass surfaces covered with the silane coupler.

Frequency dependence of capacitance of the solution in a quiescent state was measured by an LCR meter (Hewlett and Packard, 4163A) at electrical frequencies ranging from $100 \mathrm{~Hz}$ to $100 \mathrm{kHz}$. The electrodes cells used in the optical observations were used for the electrical measurement as well. External electric fields were also biased to the solution using an external bias adapter (Hewlett and Packard, 16065C) and the DC power supply.

Rheological response was measured by a rheometer with parallel plates sliding type as shown in Figure 2. The details of the rheometer were reported elsewhere. ${ }^{3,19}$

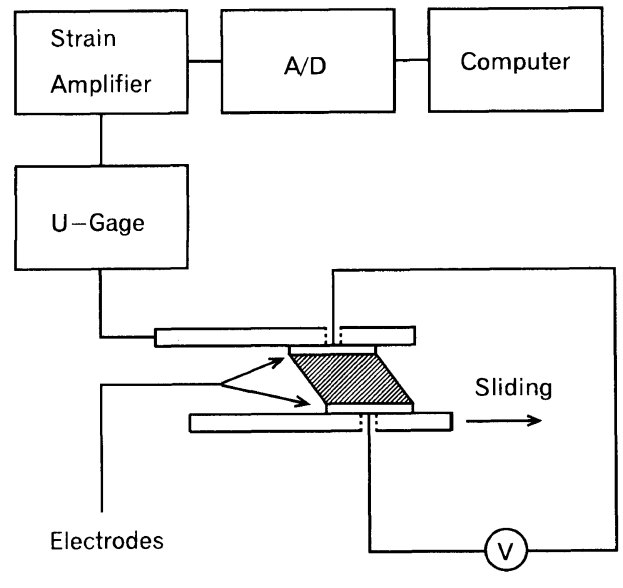

Figure 2. System for the transient measurement of stress responses.

The stress response was measured by the rheometer at a shear rate of $2.56 \mathrm{~s}^{-1}$. The solution sandwiched between the two parallel plates with a gap of $0.4 \mathrm{~mm}$ was deformed at the shear rate to be a steady flow, and subsequently it was stimulated by step-wise electric fields with amplitudes up to $3.0 \mathrm{kV} \mathrm{mm}^{-1}$. The stepwise electric fields were applied to the solution in the direction perpendicular to the parallel plates using a piezo-drive amplifier (MESS-TEK Co., M-2628) driven by a signal generator. The detected signals of stress response were digitized at a rate of $1 \mathrm{~ms}$ per Signal by a 12-bits $\mathrm{A} / \mathrm{D}$ converter, and the data were stored in a personal computer.

\section{RESULTS AND DISCUSSION}

\section{Electrical Orientation in a Quiescent State}

The PHIC solution as prepared in a cell with the electrodes of which surfaces were not covered with the silane coupler (untreated cell) under no electric fields showed dark and homogeneous figure without colorful polydomain texture in the observation of orthoscopy. At the same time, the conoscopic figure showed no isochrome but it showed a vague cross-like interference figure called an isogyre cross which indicated that the sample was a single uni-axial crystal with the optical axis perpendicular to the stage of the microscope. ${ }^{20} \mathrm{~A}$ slightly sharper but still vague isogyre cross was also seen in a cell with the electrodes of which surfaces were covered with the silane coupler (treated cell). The optical axis of PHIC is parallel to the helix axis of the molecule so that the monodomain with homeotropic orientation (perpendicular to the electrodes) was established in the present experiment. Similar results and discussion were reported by Yang and Shine ${ }^{12}$ who investigated a solution of PHIC with molecular weight as high as $1.8 \times 10^{5}$ and a narrow molecular weight distribution: Their PHIC solution as prepared exhibited colorful polydomain texture and it showed the monodomain with homeotropic texture after application of external electric fields.

The isogyre cross also became sharper when DC electric fields below $1.0 \mathrm{kV} \mathrm{mm}^{-1}$ were applied to the solution in the treated cell. And subsequently, it gradually lost its sharpness as the strength of external electric fields increased to around $1.6 \mathrm{kV} \mathrm{mm}^{-1}$. An electric field 


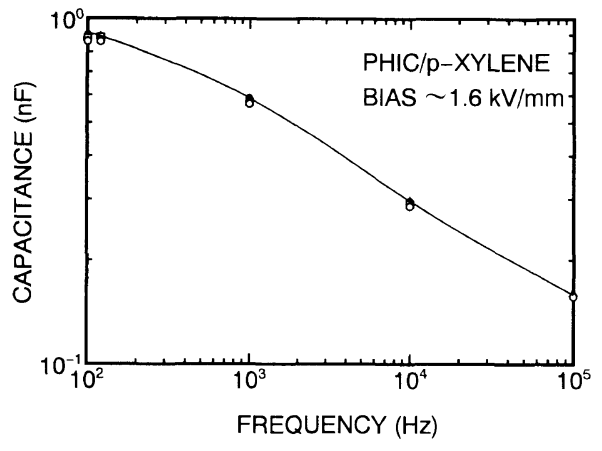

Figure 3. Capacitance of a $30 \mathrm{wt} \%$ solution of PHIC plotted against the electrical frequency. DC electric fields from 0 to $1.6 \mathrm{kV} \mathrm{mm} m^{-1}$ were biased to the solution. Solid circles represent the results without bias electric fields and the solid curve in the figure was least square fitted using the spline function and the results without bias electric fields. Open triangles, squares, and circles represent the results with bias electric fields of $0.4,0.8$, and $1.6 \mathrm{kV} \mathrm{mm}^{-1}$, respectively.

of $1.6 \mathrm{kV} \mathrm{mm}^{-1}$ induced a weak flow instability of local vortices which could be caused by the electro-hydrodynamic instability. However, the flow instability was not so remarkable as the macroscopic vortices could occur. Figure 3 shows capacitance of the PHIC solution in the treated cell (the area of the electrodes was 0.68 $\mathrm{cm}^{2}$ ) plotted against the electrical frequency. In the capacitance measurement, DC electric fields from 0 to $1.6 \mathrm{kV} \mathrm{mm}^{-1}$ were biased to the solution. The capacitance of the biased solution is almost identical to that of the solution without bias electric fields within the experimental error. Similar results of the capacitance of the biased solution as shown in Figure 3 were obtained for the biased solution in an untreated cell. The capacitance of the biased solution in the untreated cell was also almost identical to that of the solution without bias electric fields. If the macroscopic vortices were induced by the bias electric field of $1.6 \mathrm{kV} \mathrm{mm}^{-1}$, the capacitance should change remarkably at the bias electric field as discussed in the next paragraph.

Because PHIC molecule has a large permanent dipole moment along the helix axis ${ }^{17}$ and the induced dipole moment is negligible, ${ }^{18}$ the homeotropic orientation of permanent dipole moments of PHIC molecules without bias electric fields (the parallel orientation and antiparallel orientation of the dipole moments can be thought) should be randomized by the macroscopic vortices induced by the bias electric field. The (time average) vector summation of the permanent dipole moments results in zero by the randomization of the homeotropic orientation. If the homeotropic orientation consists of the parallel dipole moments, the capacitance should decrease remarkably by the macroscopic vortices. If the homeotropic orientation consists of the antiparallel dipole moments without bias electric fields, the vector summation of the dipole moments is also zero. Then, the capacitance should increase at certain bias electric fields in the process of randomization of the anti-parallel orientation where the vector summation of the dipole moments is not necessarily zero. Therefore, it is considered that the homeotropic orientation was relatively stable in the quiescent state even under bias electric fields up to $1.6 \mathrm{kV} \mathrm{mm}^{-1}$ and the electro-hydrodynamic instability was negligible from the results of

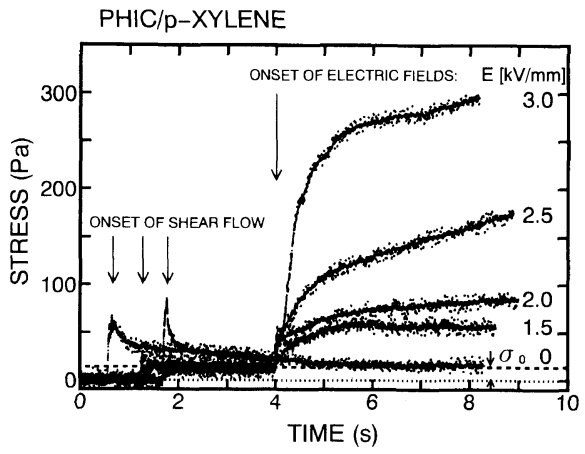

Figure 4. Transient stress responses of a $30 \mathrm{wt} \%$ solution of PHIC plotted against time. The solution was suddenly sheared at a constant shear rate of $2.56 \mathrm{~s}^{-1}$ (around the times pointed by arrows), and subsequently step-wise electric fields were applied to the solution (at the time of $4 \mathrm{~s}$ pointed by an arrow). The data of stress were smoothed by the simple moving average method among the nearest three data.

capacitance measurement. The local vortices observed by the optical microscope may be related to the flow instability of PHIC molecules with very low molecular weight components in the broad molecular weight distribution.

\section{Transient Stress Responses under Shear Flow}

Figure 4 shows transient stress responses of a $30 \mathrm{wt} \%$ solution of PHIC plotted against time. The solution was suddenly sheared at a constant shear rate of $2.56 \mathrm{~s}^{-1}$ (around the times pointed by arrows), and subsequently step-wise electric fields were applied to the solution (at the time of $4 \mathrm{~s}$ pointed by an arrow). In the present experiment, the solutions as prepared (as sandwiched between the electrodes) were used for the rheological measurements. Therefore, the orientation (or the size of anisotropic domains) of the PHIC molecules could not be controlled in advance of the onset of the shear deformation. (Although the monodomain with homeotropic orientation was established in the optical observation with a cell of $25 \mu \mathrm{m}$ gap, there could be polydomain texture in the rheological measurement with the electrodes of $0.4 \mathrm{~mm}$ gap.) In Figure 4 , the stresses at the onset of shear flow are relatively large. However, the stresses gradually decrease to reach a steady stress $\sigma_{0}$ of $13.5 \mathrm{~Pa}$. It is supposed that the PHIC molecules with homeotropic orientation immediately after onset of the shear flow were tilted toward a stable tilt angle or aligned parallel to the electrodes surfaces by the shear deformation under no electric field. The step-wise electric fields were applied when the solutions showed the steady stress. After application of step-wise electric fields, the stresses increase from the steady stress of $\sigma_{0}$ in Figure 4. In the present paper, the stress response to the stepwise electric fields is normalized by $\sigma_{0}$. The normalized ER response is defined here as $\left(\sigma(t)-\sigma_{0}\right) / \sigma_{0}$, where $\sigma(t)$ is the stress at the time after onset of the step-wise electric fields.

Figure 5 shows the normalized ER response to stepwise electric fields. In the normalized ER response, the stress overshoot can be seen at electric fields of 1.0 $\mathrm{kV} \mathrm{mm}^{-1}$ as well as $1.5 \mathrm{kV} \mathrm{mm}^{-1}$. No stress undershoot could be observed in the present experiment. The time at the maximum of the stress overshoot measured at an electric field of $1.5 \mathrm{kV} \mathrm{mm}^{-1}$ is shifted to the longer time 


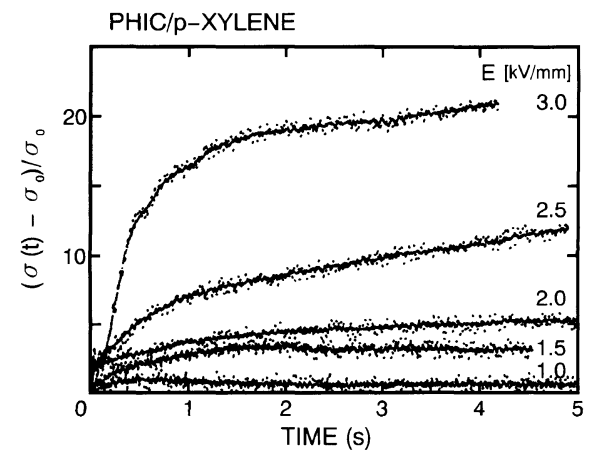

Figure 5. Normalized ER response of a $30 \mathrm{wt} \%$ solution of PHIC to step-wise electric fields after application of the electric fields. The data of the normalized ER response were also smoothed by the same method as Figure 4

than that of $1.0 \mathrm{kV} \mathrm{mm}^{-1}$. Although the reasons of the shift in time at the maximum are still unclear at the present stage, one of possible explanations based on the rod-like molecules is the polydispersity of molecular weight of our PHIC. Assuming the stress enhancement by an electric field is caused only by the changes in a tilt angle $(\theta)$ between electrodes surfaces and the director of the rod-like molecules of PHIC. The rod-like molecules which would be aligned parallel to the electrodes surfaces $(\theta=0)$ in an ideal case can be tilted toward a stable tilt angle $\theta_{0}$ sufficiently after application of an electric field. In a dynamic point of view, the molecules can be tilted toward the maximum tilt angle $\theta_{\max }\left(>\theta_{0}\right)$ after application of the step-wise electric field because of the inertia effect. And subsequently, they can be returned to the stable tilt angle $\theta_{0}$. As a rough estimation of a time scale of a stress overshoot in a sufficiently weak shear flow, the period of simple and small oscillations $(T)$ of a rod with a dipole moment $(\mu)$ under a static electric field $E$ and an unsheared situation is given to be

$$
T=2 \pi(I / \mu E)^{1 / 2}
$$

where $I\left(=\rho S l^{3} / 6\right)$ is the moment of inertia of the rod with a length $l$, the cross sectional area of the rod $\mathrm{S}$, and the density $\rho$. Because the total dipole moment of a PHIC molecule can be thought to be proportional to the length of the helix (or rod-like) molecule $\left(\mu=\mu_{0} l ; \mu_{0}\right.$ : permanent dipole moment along the helix axis per monomer unit), the eq 1 can be reduced to the eq 2 .

$$
T \propto l \cdot E^{-1 / 2}
$$

If there is an upper limit of the molecular weight (or length) of the PHIC molecules which can respond to a given electric field, it is favorable to consider that the upper limit of the molecular length increases with an increase in the electric field strength. Although the upper limit of the molecular length $l_{u}(=l(E))$ as a function of the electric field strength is unknown, it is possible to consider in eq 2 that the dependence of $l_{\mathrm{u}}$ of our PHIC on $E$ is much stronger than $E^{1 / 2}$ so that the period $T$ can be an increasing function of $E$. This discussion based on the rod-like molecules is rough and too simplified because the shear deformation and the interactions among anisotropic domains are ignored. However, the time scale of the stress overshoot in a sufficiently low shear rate can be explained qualitatively to a certain extent by the discussion above. Further, the time scale of the ER response for the rod-like molecules with large aspect ratio is expected to be much slower at a given electric field strength than that of small molecular liquid crystals because their aspect ratio is much smaller than the aspect ratio of our PHIC molecules.

The normalized transient stresses tend to approach to (normalized) steady stresses, respectively, depending on the electric field strength as shown in Figure 5. Although the steady stresses could not be necessarily measured by the sliding rheometer which limited the maximum shear strain, the constant shear rate could be relatively well assumed in the geometry. (In principle, the shear rate in the parallel plates geometry of a rotational rheometer is proportional to the distance from the center of the plates along the radial direction, ${ }^{21}$ which indicates a sample fluid is not deformed at the center, but the maximum strain is not limited in the geometry.) The maximum shear stress enhanced by the electric fields in the present experiment is in excess of 20 times the steady shear stress under no electric field. In the present experiment, the shear rate dependence of steady shear stress under an electric field was not measured. Yang and Shine ${ }^{12}$ reported that the steady shear viscosity of their PHIC solution measured at a shear rate of $0.1 \mathrm{~s}^{-1}$ was almost identical to that measured at a shear rate of $0.05 \mathrm{~s}^{-1}$ when the electric field below $0.5 \mathrm{kV} \mathrm{mm}^{-1}$ was applied to the solution. However, concentrate solutions of PHIC under an electric field with relatively low field strength may not simply show the Newtonian viscosity even at low shear rates because of the anisotropic nature of the molecules. The extensive measurements for the shear rate dependence of the steady shear viscosity under electric fields are needed to discuss the problem.

The shear stress enhancement of 20 or more times by the electric field as shown in Figure 5 is much larger than that of several times in the typical ER response of liquid crystals composed of small molecules. It is considered that the larger enhancement of the PHIC solution was caused by the larger aspect ratio of helical molecules with larger molecular weight: The rod-like molecules were preferentially tilted or aligned parallel to the electrodes surfaces in a steady shear flow before the onset of external electric fields. The tilted molecules tended to align perpendicular to the electrodes surfaces so that the permanent dipole moments of the molecules could align parallel to the external electric fields. Then, the axes of the rod-like molecules in the direction transverse to the shear flow enhanced the flow resistance. It can be supposed in an ideal case that the changes in the orientation of the rod-like molecules cause the changes in Miesowicz viscosities from $\eta_{\mathrm{a}}$ or $\eta_{\mathrm{b}}$ to $\eta_{\mathrm{c}}$ as shown in Figure 1. Therefore, the maximum ER response of the rod-like molecules can depend on the molecular weight which corresponds to the aspect ratio of the molecules. Yang and Shine ${ }^{12}$ reported the maximum ER response of thirty and several times for their PHIC solution with higher molecular weight than ours, which is consistent with the discussion above.

Further, the larger enhancement of our PHIC solution than the enhancement of liquid crystals composed of small molecules may be closely related to the tendency that the PHIC molecules preferentially aligned perpen- 
dicular to the electrodes surfaces with homeotropic orientation as shown by the optical observations. The electro-hydrodynamic instability could also affect the maximum enhancement of the ER response in the present experiment because the external electric field of $3.0 \mathrm{kV} \mathrm{mm}^{-1}$ which gave the maximum ER response was larger than the maximum strength of the bias electric fields in the optical observations and capacitance measurement. In addition, the interactions among anisotropic domains of the PHIC molecules cannot be ignored as is the case with the rheology of textured liquid crystalline polymers. ${ }^{22-24}$ The interactions among the domains can be also related to the larger stress enhancement as well as the slower time scale of the ER response for the anisotropic solution of PHIC. However, the ER response of the PHIC solution can be approximately discussed based on the Miesowicz viscosities. It is considered that the discussion based on the Miesowicz viscosities will be effective for the ER response of liquid crystals composed of rod-like molecules with large aspect ratio.

\section{CONCLUSIONS}

The homeotropic orientation was found in the solution of a commercially available poly(hexyl isocyanate) with a broad molecular weight distribution in a quiescent state under no electric fields. The homeotropic orientation was relatively stable in the quiescent state even under bias electric fields up to $1.6 \mathrm{kV} \mathrm{mm}^{-1}$ and the electro-hydrodynamic instability was considered to be negligible from the results of capacitance measurement. In the electro-rheological (ER) response of the solution measured at a constant shear rate of $2.56 \mathrm{~s}^{-1}$ by our parallel plates sliding rheometer, the stress overshoot was observed at electric fields of $1.0 \mathrm{kV} \mathrm{mm}^{-1}$ as well as $1.5 \mathrm{kV} \mathrm{mm}^{-1}$. The transient stress tended to approach to a steady stress depending on the electric field strength although the steady shear stress could not be necessarily measured by the present sliding rheometer. The maximum shear stress enhanced by the electric fields in the present experiment was in excess of 20 times the steady shear stress under no electric field. The ER response was discussed based on the Miesowicz viscosities of liquid crystals composed of rod-like molecules with large aspect ratio.

Acknowledgements. This work was partially sup- ported by The Iwatani Naoji Foundation's Research Grant. The authors also acknowledge Mr. Shikuma for the help of the GPC measurement.

\section{REFERENCES}

1. W. M. Winslow, J. Appl. Phys., 20, 1137 (1949).

2. H. Block and J. P. Kelly, J. Phys. D, 21, 1661 (1988), and references therein.

3. K. Tanaka, A. Sahashi, R. Akiyama, and K. Koyama, Phys. Rev. E, 52(4), R3325 (1995)

4. Y. Asako, S. Ono, R. Aizawa, and T. Kawakami, Polym. Prepr. Am. Chem. Soc., Div. Polym. Chem., 35(2), 352 (1994).

5. Y. Ishino, T. Maruyama, T. Ohsaki, S. Endo, T. Saito, and N. Goshima, Polym. Prep., Am. Chem. Soc., Div. Polym. Chem., 35(2), 354 (1994).

6. T. Honda, T. Sasada, and K. Kurosawa, Jpn. J. Appl. Phys., 17, 1525 (1978).

7. T. Honda, K. Kurosawa, and T. Sasada, Jpn. J. Appl. Phys., 18, 1015 (1979)

8. M. Fukumasa, K. Yoshida, S. Ohkubo, and A. Yoshizawa, Ferroelectrics, 147, 395 (1993).

9. K. Tanaka, A. Fujii, and K. Koyama, Polym. J., 24, 995 (1992).

10. A. Inoue, S. Maniwa, T. Satoh, and K. Taniguchi, Nihon Reoroji Gakkaishi (in Japanese), 23, 7 (1995); A. Inoue and S. Maniwa, J. Appl. Polym. Sci., 55, 113 (1995).

11. T. Asada, Y. Matsuo, and T. Sakurai, "Theoretical and Applied Rheology-Proceedings of XIth International Congress on Rheology (Brussels)," Vol. 2, P. Moldenaers and R. Keunings, Ed., Elsevier, Amsterdam, 1992, p. 513.

12. I-Kuan Yang and A. D. Shine, J. Rheol., 36, 1079 (1992)

13. A. Ohshima, H. Kudo, T. Sato, and A. Teramoto, Nihon Reoroji Gakkaishi (in Japanese), 22, 111 (1994).

14. M. Asano, H. Kusuoka, A. Ohshima, T. Sato, and A. Teramoto, Nihon Reoroji Gakkaishi (in Japanese), 22, 117 (1994).

15. T. Itou, H. Chikiri, A. Teramoto, and S. M. Aharoni, Polym. J., 20, 143 (1988).

16. H. Murakami, T. Norisuye, and H. Fujita, Macromolecules, 13, 345 (1980).

17. A. Bur and L. J. Fetters, Chem. Rev., 76, 728 (1976).

18. H. J. Coles, A. K. Gupta, and E. Marchal, Macromolecules, 10, 182 (1977).

19. K. Tanaka, T. Yoshida, and K. Koyama, "Electrorheological Fluids-Proceedings of 3rd International Conference on ER Fluids," R. Tao, Ed., World Scientific, Singapore, 1992, p. 289.

20. For instance, P. G. de Gennes and J. Prost, "The Physics of Liquid Crystals," 2nd ed, Oxford University Press, New York, N.Y., 1993, Chapter 3 and Chapter 5.

21. For instance, "Kouza-Reoroji" (in Japanese), The Society of Rheology, Japan, Ed., Kobunshi Kankokai, Kyoto, 1992, Chapter 2.

22. R. G. Larson and M. Doi, J. Rheol., 35(4), 539 (1991).

23. R. G. Larson and D. W. Mead, Liquid Crystals, 12(5), 751 (1992).

24. D-H. Kim, K. M. Kim, and O. O. Park, Nihon Reoroji Gakkaishi, 23, 39 (1995). 